\title{
Aliénation et inter-dit dans les romans d'Annie Ernaux?
}

\author{
Philippe Vilain \\ Université de Paris III
}

L

e plus souvent effacé au profit du thème de la déchirure sociale qui affleure en surface, la question du sexe apparaît au centre des premiers romans d'Annie Ernaux. La présence du sexe affecte d'ailleurs la plupart des paramètres textuels - lisible au niveau lexical, thématique et sémantique - et engage insidieusement le signe sexe dans un double mouvement de production et de rétention sémiotique. Le sexe apparaît à la fois comme ce qui génère l'œuvre - 1'avortement dans 1 'incipit des Armoires vides sert de moteur à 1'écriture - et comme ce qui le voue paradoxalement à la disparition.

\section{Absence et substitution du signe sexe}

Même si rien ne nous permet a priori d'affirmer que la fréquence du signe sexe est un indice méritant d'être privilégié, une brève analyse statistique mesurera efficacement son degré d'implication, d'enfouissement, dans le tissu scriptural des textes. Au niveau lexical, un relevé systematique du vocabulaire concernant le sexe et ses parties revèle ainsi la faible inscription du signe (29 occurrences + 9 désignations de parties du sexe dont clitoris, petites lèvres, vulve, penis), par rapport à mains (203 fois), yeux (118 fois), tête (34 fois), corps (96 fois), bouche (68 fois), jambes (67 fois), cheveux (66 fois), ventre (65 fois), doigts (60 fois), visage (33 fois), lèvres (30 fois). Cette pénurie d'occurrences s'avère d'autant plus surprenante qu'elle contraste avec l'impression d'une forte prégnance d'images sexuelles qui avait filtrée en première lecture. A ce niveau. nous nous en tenons aux seuls renseignements numériques comme des pistes a approfondir : il est, en effet, trop tôt pour assigner à cette défaillance du signe dans le discours une signification sociale, ces données numériques ${ }^{1}$ permettent simplement de 
constater une forme d'exclusion du signe sexe, une réticence à nommer la chose directement. La présence du sexe doit donc se chercher ailleurs. Une analyse plus profonde ne s'arrêtant plus seulement à l'inscription du signe mais s'élargissant à ses réseaux de déclinaison, prenant en compte un procédé de nomination plus vaste, nous paraît maintenant opportune.

\begin{tabular}{|c|c|c|c|c|c|c|c|c|}
\hline & \multicolumn{3}{|c|}{ 1ère période* } & \multicolumn{4}{|c|}{$2^{\mathrm{e}}$ période } & \multirow[t]{2}{*}{ Total } \\
\hline $\begin{array}{l}\quad \text { Textes } \\
\text { Nomi- } \\
\text { nation }\end{array}$ & $\begin{array}{l}\text { Les } \\
\text { Armoires } \\
\text { vides }\end{array}$ & $\begin{array}{c}\text { Ce quils } \\
\text { disent ou } \\
\text { rien }\end{array}$ & $\begin{array}{l}\text { La femme } \\
\text { gelée }\end{array}$ & La Place & $\begin{array}{l}\text { Une } \\
\text { femme }\end{array}$ & $\begin{array}{c}\text { Passion } \\
\text { simple }\end{array}$ & $\begin{array}{c}\text { Journal du } \\
\text { dehors }\end{array}$ & \\
\hline Sexe & 1 & 3 & 7 & 2 & 2 & 11 & 3 & 29 \\
\hline Parties & 1 & - & 4 & - & 1 & - & 3 & 9 \\
\hline Substituts & 14 & 12 & 11 & 1 & 1 & 1 & 1 & 41 \\
\hline Total & 16 & 15 & 22 & 3 & 4 & 12 & 7 & 79 \\
\hline
\end{tabular}

* Pour simplifier, nous ferons parfois références aux textes en termes de "période " : la première période regroupant les romans, la seconde les récits et journal.

1 Pour nous donner une idée de la faible inscription du signe sexe, nous proposons le tableau détaillé recensant le vocabulaire du corps.

\begin{tabular}{|c|c|c|c|c|c|c|c|c|}
\hline $\begin{array}{l}\quad \text { Textes } \\
\text { Partie du } \\
\text { corps }\end{array}$ & $\begin{array}{l}\text { Les } \\
\text { Armoires } \\
\text { vides }\end{array}$ & $\begin{array}{c}\text { Ce qu'ils } \\
\text { disent } \\
\text { ou rien }\end{array}$ & $\begin{array}{l}\text { La fermme } \\
\text { gelée }\end{array}$ & La Place & $\begin{array}{c}\text { Une } \\
\text { femme }\end{array}$ & $\begin{array}{l}\text { Passion } \\
\text { simple }\end{array}$ & $\begin{array}{c}\text { Journal du } \\
\text { dehors }\end{array}$ & Total \\
\hline Main(s) & 65 & 26 & 39 & 22 & 20 & 5 & 26 & 203 \\
\hline Yeux & 31 & 27 & 33 & 13 & 6 & 4 & 4 & 118 \\
\hline Tête & 34 & 20 & 24 & 12 & 7 & 5 & 16 & 118 \\
\hline Corps & 17 & 17 & 32 & 12 & 5 & 5 & 8 & 96 \\
\hline Bouche & 35 & 10 & 11 & 2 & 2 & 4 & 4 & 68 \\
\hline Jambe & 20 & 19 & 7 & 4 & 10 & 1 & 6 & 67 \\
\hline Cheveux & 16 & 9 & 15 & 7 & 10 & 2 & 7 & 66 \\
\hline Ventre & 26 & 7 & 14 & 5 & 2 & 4 & 7 & 65 \\
\hline Doigt(s) & 30 & 8 & 13 & 2 & 4 & - & 3 & 60 \\
\hline Visage & 1 & 2 & 9 & 7 & 8 & 1 & 5 & 33 \\
\hline Lèvre(s) & 12 & 2 & 5 & 5 & 3 & - & 3 & 30 \\
\hline Sexe & 1 & 3 & 7 & 2 & 2 & 11 & 3 & 29 \\
\hline $1^{\text {er total }}$ & 288 & 150 & 209 & 93 & 79 & 42 & 92 & 953 \\
\hline $2^{e}$ total $^{*}$ & +220 & +105 & +122 & +43 & +56 & +17 & +35 & +598 \\
\hline Total & 508 & 255 & 331 & 136 & 135 & 59 & 127 & 1551 \\
\hline
\end{tabular}

* Le " $2^{\mathrm{e}}$ total » prend en compte toutes les autres parties du corps énoncées dans les textes (tempes, paupières, clitoris, etc.). Par ailleurs, ces calculs quantitatifs n'excluent pas la possibilité d'une (courte) marge d'erreur. 
L'intérêt principal de ce tableau fréquentiel porte sur les importantes variations de fréquence que présentent les deux périodes. Dans les romans, la narratrice choisit de préférence le nom vulgaire ou métaphorique au détriment du nom scientifique : les substituts ( 37 fois) 1'emportent sur la nomination directe. Dans les récits, la fréquence accrue de la nomination directe (22 fois) engendre à l'inverse une dé-substitution ( 4 fois). La narratrice de Passion simple et de Journal du dehors ne répugne plus du tout à nommer la chose par son nom (clitoris, petites lèvres, etc...) et présente un sexe, quasiment fonctionnel, décanté de sa flamboyance lyrique. Mis en roman, le signe sexe affronte une menace d'entropie, d'épuisement énergétique, qui se projette en un effondrement net du signe-référent. Cette perte affleure symptomatiquement dans une série de substitution, de dérives : le sexe masculin se décline en zizi, zézette, chose, titite, baisette, bite, trique, machine, une, truc, queue, ça, endroit, misère, couilles; le sexe feminin en quat'sous, catsous, petits bonbons, carabis, carabo, ton, celui-là, crougnougnous, mystère de mou, trou humide, petite maison rouge, secret, notre, petit couloir au volet fermé, bête, moteur, trou humide à pipi, creux muet et invisible. Il faut lire le sexe là où il n'y a que des caches, des pagnes. La substitution opère un brouillage du signe afin que l'infigrurable s'inscrive en creux de la représentation, au lieu-même où la nomination directe abdique. Tendance qui permettrait de signifier que le lexique mime l'accord avec le référent : en effet, au sexe débouté, "bousillé " présente dans Les Armoires vides correspond un vocabulaire résolument incisif et violent, cru et provocateur; à 1'innocence des premiers attouchements sexuels de l'enfance correspond un langage métaphorique masqué sous un jeu de significations plus complexe. Sujet à de nombreuses réticences ou à une forte production imagée, dépouillé de sa gangue verbale ou recouvert d'un épiderme métaphorique, le sexe se définit dès lors comme un enjeu social du texte, le lieu stratégique des tensions entre le texte et le hors-texte, des conflits entre le dit et le non-dit.

\section{Le discours oblique et l'inter-dit}

Chez les trois jeunes narratrices des Armoires vides, Ce quils disent ou rien et La Femme gelée, le sexe appartient au domaine de 1'interdit, c'est la raison pour laquelle it ne peut pas encore se verbaliser, il se dissimule sous " des mots murmurés comme au confessional » (Les Armoires vides, 31) et n'existe que de façon latente: "Pas beaucoup de noms, on ne soupçonnait pas qu'il puisse même y en avoir de sérieux dans le dictionnaire pour ces choses "? (La Femme gelée, 42) Les narratrices se réferent à la Chose 
sexuelle, aux premieres règles, par le "ça ", "faire ça ", " être comme ça ».(La Femme gelée, 42) Elles proponent, par conséquent, une vision détournée du sexuel, une vision médiatisée par tout un ensemble de procédés littéraires qui font entrevoir 1'irréprésentabilité du sexe lié à la condamnation de l'apparition directe : "Ninise, baisse le capot on voit le moteur ". (Les Armoires vides, 31) Même si les frontières ne sont pas toujours évidentes à tracer entre tous ces procédés, nous dégagerons quelques figures réthoriques inhérente à cette vision indirecte.

La brève scène de l'avortement au début des Armoires vides offre un modèle caractéristique du discours oblique. Indirectement désigné (quat'sous et petits bonbons), le sexe n'est jamais montré, il est allusivement localisé par la narratrice " quelque part au bas du ventre » ou " entre (l)es jambes ». L'endroit indiqué suggère un autre endroit qu'il n'exprime pas et dont la pleine intelligence suppose la perception d'un rapport intertextuel entre ce qui n'est pas désigné et la suite du texte. Quelques lignes plus has, l'inscription du signe sexe est réduite de manière spectaculaire, le signe se condense comme s'il subissait les affres dune excision verbale, it se reduit aux deux lettres d'un autre signe, son pronom personnel : « Je me demande s'il resservira un jour ", puis à une simple lettre " tu l'abîmerais ", ou bien il est mutilé, coupé en deux, "quat'sous ». Le langage opère d'autorité une réduction de la prononciation - comme pour signifier son peu de valeur et 1'énonciation du sexe doit être écourtée : "Rien qu'à nommer, c'était drôle, la machine, la baisette, une pour abréger ». (La Femme gelée, 43) L'ellipse peut enfin avoir lieu et continuer de filer ce discours sous entendu : 'J'ai engueulé la vieille, qui bourrait d'ouate pour faire tenir ». Précisément là où le lecteur était en droit d'attendre un complément d'objet direct : j'ai engueulé la vieille qui (le) bourrait (le sexe) d'ouate pour faire tenir. L'éviction est ici plus nette qu'ailleurs, elle aboutit à une forme elliptique extrême, où la concision et la densité conduisent progressivement à son exclusion, à sa disparition complète. Le discours devient syncope : " Il ne faut pas toucher ton quat'sous, tu l'abîmerais... laisse-moi embrasser les petits bonbons, là, entre les lèvres... Crocheté, bousillé, colmate ". Cette réticence qui relève de la fonction émotive a ici un double statut : d'une part, elle change l'objet-même du discours, détourne son orientation (" laisse-moi embrasser... ", intervention de la scène passée dans le présent) pour la reprendre une phrase plus loin, à l'endroit de la suspension, là où elle l'avait laissé en plan (procédé typique de l'aposiopèse); d'autre part, ce détournement est illusoire puisque la réticence est ici le moyen de se remémorer les préludes de 1'acte sexuel qui ont entrainé la narratrice dans 1'appartement de la faiseuse d'ange et, en 
quelque sorte, de relier implicitement 1'avortement à la faute originelle : "Ce sera pareil, je ne peux pas m'empêcher de confondre les deux. C'était dans la deux-chevaux, ce sera peut-être ici, à la Cité. La vieille m'a demandé si j'avais perdu beaucoup de sang au dépucelage. Ecarter les cuisses, pareil ". (Les Armoires vides, 171)

Le spectacle du sexe donne peu àvoir, il n'est àproprement parler jamais nommé et très peu décrit - quoique le recit multipliant les images implicitement liées au sexe annonce, prépare, en fasse attendre un tableau détaillé qui serait, peut-être, le pendant de L'Origine $d u$ monde ${ }^{2}$, le tableau de Courbet, en écriture. Le paradoxe peut d'ailleurs surprendre : tel un zoom de camera, la description se focalise dès les premières lignes sur I'avortement, entraine le lecteur dans le vif de l'action, in medias res, mais toujours sans s'attarder sur le sexe et prenant soin de décrire systématiquement son entour, l'avant ("Ca vous chauffera une minute. Juste le temps d'enfoncer ») et I'après ("Après elle m'a fait boire un café pour nous remonter "). Le sexe gît dans cet espace entre-deux, dans les blancs et les creux de la description, simplement désigné par la douleur résorbée au milieu du discours : "Atroce ». La technique ernalienne consiste ici à créer, à susciter puis à frustrer le désir de voir. Tout se passe en effet comme si la vision du sexe imposait un détour du langage, comme si la Chose devait se métamorphoser en autre chose ${ }^{3}$. Si ce détournement permanent permet à la fois d'esquiver sa représentation dans un déplacement d'images et de discourir sur cet objet sans y compromettre sa " dignité », il bute sur un paradoxe, à savoir que même évité le sexe resiste. suspend le dire en faisant appel àun autre mode de signifance. Il n'est pas davantage décrit, mais constamment re-nommé, refiguré, déplacé, presque dans une dimension anaphorique, qui inscrit au cœur de cette substitution pléthorique sa propre irréductibilité.

Ce detournement nous indique cependant que le degoûtant et le sale ne sont peut-être pas contenus dans la Chose elle-même, mais dans les termes qui la caractérisent et que les parents ne prononcent jamais. De fait, le sexe ne se constitue pas comme un indicible mais comme un dicible qui

2 Dans Passion simple, (p. 50). Annie Ernaux regrette « qu'il n'existe pas, peint par une femme, un tableau provoquant autant d'émotion indicible que la toile de Courbet montrant au premier plan le sexe offert d'une femme couchée, au visage invisible, et qui a pour titre L'Origine du monde ".

3 Par un procédé analogue, l'apparition des premières règles sont I'occasion de " litotes honteuses " : " la visite de la Tante Rose ", « les anglais ont débarqué ", " indisposée ", " handicapée ".

Vilain 55 
s'offre à une nomination circonscrivant la part dechue du langage dominé. Comme si la narratrice, d'extraction populaire, utilisait les ressources du language de la classe dominante pour masquer l'impropreté d'un sexe liée à sa classe sociale : "Quelque chose de poisseux et d'impur m'entoure définitivement, lié à mes différences, à mon milieu ». (Les Armoires vides, 67) En ce sens. la vulgarité de la nomination n'est peut-être rien d'autre ICI que l'emploi des mots vulgaires, du vulgus, car la décheance du sexe est inexctricablement liée à son appartenance sociale, c'est en quelque sorte l'aliénation, la marque de classe d'un language dominé qui, par essence, est déjà obscenité avant d'être énoncé : "catsous, dessous, souillé. Sale à cacher ". (La Femme gelée, 40) Cette tentative d'éradication systématique du sexe redouble le sens de l'aliénation des narratrices. Son comportement s'inscrit dans un contexte marqué par les contraintes du système catholique : "A leur tour, la vierge, les saints, 1'Eglise adorée condamnent jusqu'à mes pensées ». (Les Armoires vides, 66) Ces contraintes ne manquent pas de faire ressortir les tares réputées propres à la nature féminine et s'érigent en principes : la souillure ("j'étais de nouveau sale et impure ») (Les Armoires vides, 143), la sexualité tabou ("C'etait dangereux à leur idée comme de se toucher le carabi ") Ce quills disent ou rien, 65, le plaisir coupable ("Toucher, la belle blague, c'est pas ça qui est mal, c'est le plaisir ") Ce qu'ils disent ou rien, 100, le chatiment ("La punition. La vraie, enfin arrivée... ") (Les Armoires vides, 122). Tous ces principes sont sourcés dans le dogme du péché originel, clef de voûte du monde chrétien et de la présentation du sexe dans les romans ernaliens fondés, en partie, sur la problématique de la Faute. L'idéologie chrétienne est comme infusée dans le comportement des narratrices, dans leur silence et leurs réticences, qui sont autant d'indices susceptibles de nous renseigner sur la condition d'une jeune fille vivant en milieu populaire dans les années 50. A travers la sexualité, ces romans fournissent une échelle assez caractéritique de cette période. Ainsi, la piété religieuse de la mère, la vie en milieu populaire dans le contexte de l'immédiat après-guerre, tous ces éléments ont, semble-t-i1, déterminé la sexualité de narratrices qui ont successivement intériorisées les normes et les valeurs de cette société.

\section{La recherche du " corps glorieux »}

Par le fait dune censure familiale et sociale, 1'obsession du sexe reste encore indicible en langage non transposé. De même, le discours amoureux des parents n'était possible que sous la forme d'allusions : « II lui disait souvent des choses ordinaires, mais en la regardant fixement, elle baissait les veux et

\section{LittéRéalité}


s'empechait de rire ". Et, toute expression de leurs sentiments se trouvait occultée, les parents n'avaient pas " de caresses ni de gestes tendres l'un pour 1'autre ". ( La Place, 37) Le sexe n'a jamais accès directement à la représentation, pour se rendre présentable, il n'apparaît que médiatisé sous la forme d'images ou neutralisé par un ensemble d'effets de texte, toujours résorbé dans un "faire signe ». L'écriture ne 1'intègre jamais tel quel mais le regénère en le recouvrant d'une patine métaphorique. Son existence dans le texte est soumise à une forte pression fantasmatique que la nourriture, symboliquement, est chargée de préfigurer, inversant les valeurs et s'appuyant sur la capacité de transmutation poétique propre à la langue. Désormais, la nourriture participe à l'émancipation sexuelle des narratrices et sert par là-même de support à tout un imaginaire lié à la sexualite. Le sexe est place sous le signe du fantasme, du desir et de 1'appetence. 11 se presente sous la la forme d'un bonheur de la sensation ou chaque élément revêt un sens éminemment symbolique. Pour s'en persuader, it suffit de mettre à 1'épreuve, ce passage tiré des Armoires vides.

\begin{abstract}
Puiser à pleines mains dans les bonbons roses, les pastilles de menthe, en croquer cinq ou six à la fois, s'emplir la gorge de cete liqueur des parfums mélés [...] Sentir la saveur m’imprégner, me submerger... Mes fringales, j’ai de quoi les appaiser à profusion [...] Un bonbon par-ci par-là. Mottes de beurre que je dépichce, larnelles de fromage taillées de biais au couteau, [...] molles et jaunes au bout des doigts. Même la moutarde dans les grands pots. J’y enfonce énergiquement la cuiller de bois pour voir me résister une marée verdâtre qui picote les ycux et les 1èvres. Cubes de viandox enrobés de papier doré comme des bonbons de luxe, salés, brûlants au palais. Régimes de bananes en vagues douces. En hiver, les oranges empilées dans les cageots, leur odeur se mélange à celle du moisi des murs, les petits Jésus de guimauve qu'on dirait fermes et qui s'écrasent, élastiques, entre les dents, le Père Noël avec un ruban rouge au cou, que je tourne et retourne avant de lui sectionner son ventre creux et vide. Je ne résistais pas au rouge tendre des cerises confites. sous le sachet de cellophane qui muitiplie leurs reflets (Les Armoires vides, 31)
\end{abstract}

Ces images ont le caractère plein, absolu, de 1'accomplissement sensuel, clairement traduit par les verbes imprégner, sentir, submerger, apaiser. Ici. l'utilisation du pluriel, la démultiplication des images, leurs " reflets », indiquent la plongée dans un monde irréel matérialisé par le café-épicerie à partir duquel vont résurgir les souvenirs d'enfance. Car le véritable idéal plonge dans cette enfance, disjointe de réel, où a posteriori 
la nourriture et le sexuel cohabitent, font partie d'un même tableau dont chaque élément semble exprimer le désir et le rêve. C'est dans l'abondance que la nourriture devient accessible et apparaît comme une offrande : "Je ne connais que la profusion, tout ce qui me manque est offert dans les rayons, en boîtes, en paquets, en vrac, peux toucher à tout, entamer, grignoter, épignoler tout ». (Les Armoires vides, 32) Nous le pressentons, la nourriture fonctionne, d'une part, comme un élément religieux, mais renversé, qui fait du sexuel un graal dissimulé, un idéal à conquérir, d'autre part, comme élément autobiographique. Elle permet métaphoriquement d'approcher la Chose innommable, irreprésentable, de la conquérir et de la fixer, c'est-à-dire à la fois de se saisir de la Chose et d'arrêter le temps et 1'espace dont elle s'entoure, comme le ferait une nature morte en peinture ou bien une photographie. Le plaisir de la nourriture, notamment des confiseries et des sucreries, tient des retrouvailles de la narratrice avec un certain ordre sensoriel, latent. pius, plus vif d'etre secondairement inscrit, qui permet à la fois de retrouver "la langue d'un pays imaginaire " (Les Armoires vides, 78), la langue imagée de la petite enfance, et de retrouver l'intégrité corporelle du désir par le souvenir, par le langage fait de sensations tout autant que de mots.

Scène primitive. Fantasmatique, se présente comme le produit dune activité semi-onirique. Les mots sont limpides : "L'épicerie, la seconde partie du monde après le café, abondante, variée, croulante de plaisirs » (Les Armoires vides, 32), (le pluriel placé sur "plaisirs " en souligne la polysémie). Le sexuel étant proscrit et inaccessible, le désir, par transfert, se porte sur la nourriture seule capable de reconduire la narratrice dans le paradis de 1'enfance, de remonter en même temps à la source de 1'interdiction, en somme, d'incarner 1'idéal. A travers les confiseries et les fruits, le je court à rebours vers le plaisir et surtout vers un espace et un temps mythiques, confondus. Pour nous résumer, le je se précipite dans un mouvement de quête très complexe : après le péché qui a conduit à l"avortement, il s'agit pour Denise Lesur de trouver le salut, la régéneration par la recomposition d'un cadre spatio-temporel disparu, que la nourriture peut incarner très symboliquement, seule représentation vivante qui la rattache au monde de 1'enfance. C'est tout ensemble une quête de la vie, de 1'amour, de 1'enfance, du sexe, de 1'innocence. C'est encore la quête d'une identité et celle d'un temps mythique qui aurait fixé une fois pour toutes ses sensations.

Faisant dériver le champ sémantique de la sensation vers celui de l'imaginaire et du retour aux ongines, la nourriture joue un rôle d'embrayeur, de 
catalyseur : elle favorise la quête du sexuel, sans que soit mises en jeu la pensée consciente et la volonté. Role qui n'est pas sans rappeler cette Petite Madeleine trempée dans une infusion de thé d'où va surgir "1'édifice immense du souvenir " de Proust. Par ces fonctions, le café-épicerie " nourrit " la mémoire et devient le lieu-même de la métaphore, lieu mythique de la remise en scène de 1'enfance, ou se répète un déjà-là. Le souvenir d'enfance permet de retrouver dans la véracité des faits et la fidélité des sensations une vérité, un mot, de reconstruction romanesque : "Moi, Denise Lesur, moi ". (Les Armoires vides, 47) La narration défiant et s'arrêtant sur des moments fixés par la sensation retrouvée, la succession de métaphores sexuelles relève d'une esthétique de la fragmentation par ses ruptures multiples, qui reconstruit, bribes par bribes, un fantasme. Tout le long, le texte établit un lien sémantique entre les sensations du corps et une explication soudain découverte. Retrouver la sexualité, même déguisée, du passé, c'est se retrouver, retrouver des sensations, des désirs liés par le souvenir de sa propre chair. C'est pourquoi les mots de la narratrice se substituent souvent à la voix de 1'enfant. Cette polvphonie participe d'une entreprise de recréation du passé, d'une quête de la sensation disparue. Ainsi, la narratrice et la jeune fille, le je de l'enfance et le moi du présent, revivent ensemble dans les romans. En ce sens, il s'agit d'un retour à l'origine, origine de soi dans le nid de voix et de chair ou la reconnaissance du sexuel est inséparable d'une connaissance de soi.

\section{Jouissance refoulée/transgression substituée}

La nourriture rassemble les mêmes caracténistiques que le sexuel, le même registre lexical de l'interdiction en assure le lien : "la tentation toujours satisfaite, mais en douce "; " un regard à droite et à gauche pour voir si personne ne vient, et deux ou trois fruits collants vont juter délicieusement sur ma lamgue ». (Les Armoires vides, 31) Comme le sexuel, la nourriture est un moyen de compenser les frustrations subies sur le plan social, un « ersatz » générateur du même sentiment de culpabilité. Par conséquent, la nourriture ne peut être, par mimetisme, que de l'ordre du caché, de 1'interdit : "Je file avec mes poignées de reglisses logée rapidement sous ma jupe, dans la culotte, le seul endroit ou on n'ira pas farfouiller " (Les Armoires vides, 32) ou " Il ne faut jamais montrer qu'on rêve d'un bout de saucisson ». (Les Armoires vides, 30) Le travail sur le sens implique une relation double, polysemique avec l'objet qu'ils décrivent, à savoir, ici, le système d'expression d'une réalité qui n'a rien de sexuel dans ses principes 
mais que nous distinguons au moven de termes exprimés dans le vocabulaire de la nourriture. A ce titre, les deux ensembles sémiques présentent un champ commun :

" la tentation toujours satisfaite"

Sexuel "ne pas se faire prendre " Manger/Nourriture

comparé "rêve dun bout de saucisson " comparant

L'interséction sémique des deux langages - lieu de co-possession de sèmes - modifie le contenu sémantique du comparé sexuel et le substitue par addition/suppression : le sexuel devient comestible. Les verbes reliant les deux parties assurent le passage de la transposition (trembler, trotter, sortir, oser, fendre, sentir, éplucher, sucer, juter, jaillir, livrer). Passage justifié par "ces mots a double sens " que la narratrice " n'osait pas employer à cause du sens sale ". (Ce quills disent ou rien, 91) Ainsi, en tentant de substituer l'image du sexe à celle de la nourriture, les narratrices filent une métaphore sans cesse reprise et reincarnée de leurs désirs inconscients, se mettent à exhiber les images interdites que leur conscience a longtemps refoulées : "Rien à faire, moins je veux y penser et plus j'y pense ». (Les Armoires vides, 88)

\section{L'image de la fellation}

" jc frotte mes lèvres rentrées - c'est la première fois- à quelque chose de mou, d'odorant, de rugueux "; " chaud aux cuisses d'y penser, bouche ouverte, collante de sucre... "; "les spirales de réglisse Zen, les bâtons a sucer s'illuminent ", " me livrer sans autre goût au plaisir cremeux. parfumé "; " sucer la crème accrochée aux fils râpeux de la serviette "; " 'J'ai le morceau dans la bouche, plus amer que je l'aurais cru "; "ma bouche gonflée de crème et d'hosties imaginaires "; " oser fendre la peau plissée de ma poire au jus "; " on a mangé des croustillons, des types nous ont suivies, c'est bon les croustillons? [...] Gabrielle m'a jeté un oil en coin voir si j'avais compris [...]. Ca recommençait chaque fois qu'on portait un croustillon à la bouche " : « sucer avec délice »; « Brigitte suçotait son herbe et riait à petits coups réguliers, du haut du palais. Il était en short, sans chemise, je voyais ses muscles, sa peau ». 


\section{L'image de l'éjaculation}

« il sort [...] une gouttelette sucrée quand j'enfonce l'ongle »; " au creux de la main la laitance crémeuse et fragile ", "piqué de clous de girofle, d'oignons, fondants, acides sous la langue "; « "les mains... c'était plein de jus, j'avais cru que c'était de la pisse, c'est à l'odeur que j'ai senti la différence "; « filets de mousse blanche, elle versait la goutte sur le fond de sucre collé de la tasse ", " j’ai les mains perlées de sucre ", " tremper le doigt dans la pâte, $[\ldots]$ nous raclons le fond crémeux "; « mes mains tremblaient pour élucher la pêche, du jus gouttait le long de mon poignet "; « un sexe immense, une formidable envie, un écoulement de sang et de sperme ».

\section{Limage de la masturbation}

"brouillard rose, gigantesque fleur de mains épanouies entre les jambes, chou monté qui la cache toute, et elle, là-dessous, protégée, immobile, heureuse "; " Je touchais, paumes collés aux fromages, à la surface gluante des baquets d'eau de lessive vieille de cinq jours, doigts dégoulinants de confiture, petits fouineurs... $n^{4}$.

Il est clair que ces métaphores valent principalement par leur réitération, qui permet d'exhiber souterrainement les images censurées. Le comparant se prolonge en aval des romans, se met à produire. Ces métaphores, en multipliant les signifiés du sexuel, en les propageant dans les romans, transforment la chair defendue en un objet détourné de jouissance, comme si la capacité é jouir érotiquement se trouvait déportée dans l'imagiinaire, dans la fiction, à partir de laquelle la narratrice peut se fantasmer : « Je sens d'avance ma tricherie, je vais valoriser tout ce qui me paraissait alors si moche, mon corps réel, le plaisir ». (La Femme gelée, 62) Loofftce érotico-littéraire célèbre le mystere de l'incarnation du sexe qưil s'agit de consommer par l'imaginaire. De fait, l'emploi audacieux, obsessionnel, de la métaphore permet non seulement aux narratrices de s'affranchir du poids de l'idéologie catholique et, surtout, de trouver un équivalent de la sensation d'orgasme. La conversion du négatif en positif a bien fonctionné puisque le sexe n'est plus associé à des éléments répulsifs,

4 Afin d'éviter la surabondance de notes inutiles, nous regroupons ces notes, respectivement, dans l'ordre de leur apparition : fellation : p. 20. 30. 30, 39, 40, 41, 40, 49 (Les Armoires vides) et p. 55, 84 (La Femme gelée) : éjaculation: p. 44, 48, 48, 88 (Les Armoires vides); p. 12, 23, 23, 84, 71 (La Femme gelée); masturbation : p. 30, 49 (Les Armoires vides). 
mais, au contraire, à des éléments constitutifs du plaisir. Sexe et écriture ne sont donc plus dans un rapport simplement représentatif mais dans un rapport métaphorique qui convertit la negativité du sexuel en image positive. La nourriture, elle, participe de ce raturage du sexe sale et impur de l'enfance, et opère une médiation nécessaire entre l'objet et sa représentation. Le sexe et son signe ne peuvent coexister; cependant, la dimension que les narratrices veulent conférer à ces images est celle des signes, du plaisir des mots : pour exister et produire de l'érotique, ces images exigent un redoublement de sens par jeu de substitution en même temps que la privation de l'objet (qui suscite, en contre partie, l'adoration du signe). Métaphore qui, en verbalisant les images interdites de l'inconscient, permet la figurabilité du sexe, c'est-à-dire de repousser les limites du langage, d'assigner é la nourriture un autre sens, et de lui faire dire plus qu'elle ne peut dire ou figurer. Déguisant de la sorte le lexique sexuel, ces romans mettent en scène un déplacement de sens que nous schématisons comme suit :

Gourmandise sexuelle Gourmandise nutritionnelle (négatif) (positif)

Inconsciemment, les narratrices déplacent sur la nourriture le sens latent de l'interdiction, transferent sur la matière nutritive la jouissance ressentie lors des premiers fantasmes. Ce transfert est assumé, par la métaphore, systeme pratique de substitution, car la sublimation du sexe par la nourriture est de nature purement transgressive, transfert du péché originel en péché capital, dont la gourmandise est l'enjeu. Il y a une inversion des signes de la catholicité en signes athés puisque la subversion du péché autorise une sorte de fornication imaginaire et légale. La métaphore prend toute sa signification par rapport à cette rature du sens catholique. Il s'agit cependant dune représntation fictive de la transgression car le déir sexuel, résorbé dans une combinatoire de possibles et inscrit dans l'ordre du concevable et du dicible, est bel et bien dénié dans sa réalité, dans son statut de pure image.

L'aliénation par rapport au sexe se marque précisement par cette déprésentation constante, à savoir, par son caractère déviant et oblique, où tout un ensemble de figures réthoriques parviennent à restaurer, à maintenir 1'image du sexe dans un immédiat visible mais retrécit, dans une instantanéïté qui apparaît comme un leurre. La muitiplicité des métaphores et des substituts qui s'entrecroisent dans les romans entoure le signe sexe d'images instables et circulaires dont le va-et-vient de signifiance 
s'abolit en se refractant, comme dans un palais de glaces ou des dizaines de miroirs disposés les uns en face des autres ne reflèteraient que le vide. Dans la forme qui se représente, le sexe fait constamment retour, joue l'effet boomerang, comme signifiant déplacé; tandis que la nourriture metonymise le sexe, la métaphore réalise le sexe signifé. Par là se désigne une certaine perte de la signifiance, une atrophie verbale d'un sexe mutilé qui, dans les récits, n'apparaîtra plus que dans sa fonctionnalité, restreint dans ses emplois organiques, dans l'instrumentalité d'un sexe-objet. Cette configuration de 1'aliénation renvoie, sans doute, à une représentation latente du sexe dans i'enfance de l'auteur, s'affirmant comme une donnée du hors-texte que les trois romans absorbent et dont ils s'accommodent. Nous l'avons dit, la présentation du sexe s'inscrit dans une zone de réticence et de non-dit qui entre en collision avec l'idéologie catholique de l'époque, en temoigne le silence exercé autour de la sexuaiité : " Il faut dire qu'il y a eu un blanc dans son mode d'emploi de la vie ". (La Femme gelée, 40) Dans ce cas, la substitution du signe pourrait bien être le reflet du silence imposé aux narratrices, dont elle ne sont évidemment pas responsables, mais prisonnières et complices dune société ou parler de la sexualité est déjà " considérée comme le début du vice " (Une Femme, 60) Le svsteme catholique et 1 'interdit jouent de concert : le premier fondé sur des valeurs "répressives » se renercute au niveau de la textualisation du sexe dans les romans, le second met en scene des conditions sociales qui en assurent sa figuration, et engage du même coup, articulé sur ces conditions, tout un ensemble de procédés réthoriques qui reproduisent symboliquement leur propre logique d'interdiction, leur propre loi du silence. A cette fin, le sexe apparaît comme un graal inaccessible, inter-dit, une sorte de Dieu caché qui doit se chercher entre les lignes. 